\title{
Introduction to selected papers from GIW/InCoB 2015
}

\author{
Published 3 November 2015
}

This issue of JBCB features several articles from GIW/InCoB 2015, held in Tokyo, Japan on 9-11 September 2015. GIW/InCoB 2015 was remarkable as the first joint conference between the two major bioinformatics conferences in Asia, viz. GIW (International Conference on Genome Informatics) and InCoB (International Conference on Bioinformatics). The collaboration was reflected in a broad mixture of theoretical and applied work presented by participants from throughout Asia and the world. The conference was highly participatory, with approximately 200 participants presenting 78 poster and 81 oral presentations, including 51 original research presentations to be published in a portfolio of journals, such as this issue of JBCB.

Three papers have been selected to be published in this issue of JBCB. The papers addressed a number of important issues in bioinformatics. Existing computational approaches for analyzing gene regulations assume that the models are the same throughout whole time-course experiments. However, this assumption makes it difficult to study the transient changes at each time point for a dynamic system. Takenaka et al. proposed a score that can be used to analyze gene regulations at each time point to tackle this problem. Han et al., on the other hand, tried to solve the problem of automatic protein function annotation. They proposed a sparse Markov chain-based semi-supervised MIML method to capture the affinity between proteins for a more accurate annotation. Experimental results show that their approach performed better than other state-of-the-art MIML learning algorithms. Nguyen et al. also worked on protein function annotation, but focused on enzymes. Instead of using sequence similarity, they proposed to use functional domain architecture to score the association between domain families and enzyme families. The tool they developed, EnzDP, is a valuable tool for enzyme annotation and metabolic network reconstruction.

\section{References}

1. Han C, Chen J, Wu Q, Mu S, Min H, Sparse Markov chain based semi-supervised multiinstance multi-label method for protein function prediction, Journal of Bioinformatics and Computational Biology 13(5):1543001, 2015. 
2. Nguyen NN, Srihari S, Leong HW, Chong KF, EnzDP: Improved enzyme annotation for metabolic network reconstruction based on domain composition profiles, Journal of Bioinformatics and Computational Biology 13(5):1543003, 2015.

3. Takenaka Y, Seno S, Matsuda H, Detecting shifts in gene regulatory networks during timecourse experiments at single-time-point temporal resolution, Journal of Bioinformatics and Computational Biology 13(5):1543002, 2015.

GIW/InCoB 2015 co-chairs

Paul Horton

Christian Schönbach

Shoba Ranganathan

Siu Ming Yiu 